\title{
Real Time Intelligent Thermal Analysis Approach for Early Diagnosis of Breast Cancer
}

\author{
Pragati Kapoor \\ Astt. Professor \\ Lingaya's University \\ Faridabad, Haryana
}

\author{
Ekta Bhayana \\ Lecturer \\ Apeejay College of Engg. \\ Sohna, Haryana
}

\author{
Dr. S.V.A.V Prasad \\ Professor \\ Lingaya's University \\ Faridabad, Haryana
}

\begin{abstract}
This paper proposes an approach for early diagnosis of breast cancer using high definition thermal imaging. Breast cancer is a common and dreadful disease in women. The advanced computer technology available today can be utilized to automate the analysis and assist in decision making.This paper attempts to identify the use of infrared thermal imaging or thermography of the breast as a non-invasive adjunctive diagnostic methodology for breast cancer. Thermal imaging, as a test of physiology can detect subtle changes in breast temperature that indicate a variety of breast diseases and abnormalities.
\end{abstract}

\section{Keywords}

Breast cancer, high definition thermal imaging, thermography, non-invasive, adjunctive diagnostic methodology.

\section{INTRODUCTION}

Breast cancer has been known for decades to be the most common type of cancer among women. In United States alone, it is estimated that there are 1 million women with undetected breast cancer and 45,000 women die per year (Diakides \& Diakides, 2003). This high death rate has stimulated extensive researches in breast cancer detection and treatment. Recent studies have determined that the key to breast cancer survival rests upon its earliest detection possible.

However, mammography the most widely employed detection method is not as effective for women with dense or surgically altered breasts, or women aged 40 and younger. Furthermore, there is concern regarding the risk of ionizing radiation and patients complain of discomfort due to the high compression of breasts. In searching for other imaging techniques to complement mammography, thermography can emerge as a potential method to improve overall detection efficiency.

Advances in camera technologies and reduced equipment costs have lead to an increased interest in the application of thermography in the medical field. Thermal imaging uses a camera with sensitivities in the infrared to provide a picture of the temperature distribution of the human body. It is a non-invasive, non contact, passive, radiation free technique that can also be used in combination with anatomical investigations based on X-rays and often reveals problems when the anatomy is otherwise normal.

\section{THERMAL RADIATION THEORY}

\subsection{Planck radiation law}

Any object whose temperature is above absolute zero Kelvin emits radiation at a rate and with a distribution of wavelengths.

Human skin emits IR radiation mainly in the range of 2-20 micrometer wavelength. With the application of Plank's equation and Wien's law, it is found that approximately $90 \%$ of the emitted IR radiation in humans is in the longer wavelengths (6-14 micrometer)

\subsection{Infrared Thermography}

IR thermography makes use of a thermal imager to detect the IR radiation and measure the heat pattern of the object surface or human skin. It is passive in nature as it will not emit any harmful radiation or subject the patient to any risk. Hence many consider thermography as a physiological test (i.e. normal and abnormal physiologic functions of the nervous, vascular and muscular system and local inflammatory processes are imaged) compared to anatomical tests such as mammography.

Other advantage of thermography include high portability and real time imaging, which makes it possible for the data to be recorded in computer for processing. Clinical thermogram resembles the anatomic area under study which represents the patient's skin surface temperatures creating thermal patterns.

The temperature is an illustration of many colors each of which indicate a particular temperature. It is worthy to note that however false colors do not represents a particular temperature. They just indicate a threshold of temperature, meaning a false color band is representing all temperature values above the threshold and below the threshold of the adjacent color band.Fig 1 shows a basic setup of a typical thermal imager.

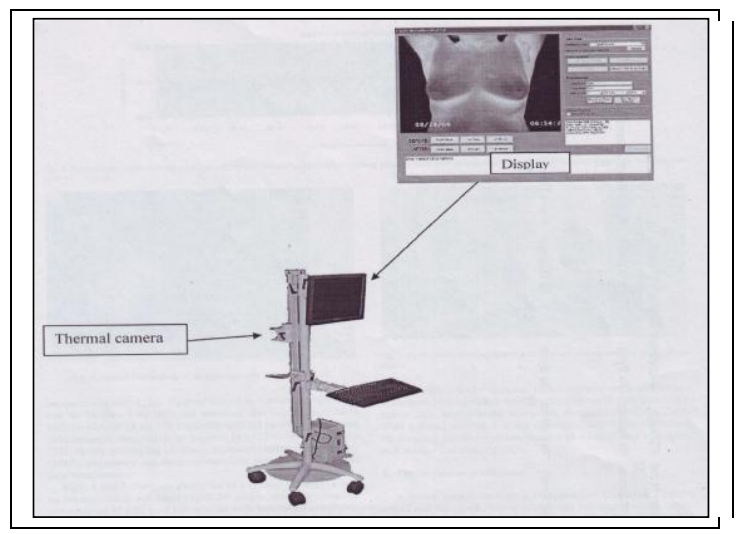


Fig1. Thermal imager setup (courtesy of www.spiritsolutions.us)

While IR radiation is invisible to human eye, it can be detected and displayed by special IR cameras. The thermal imager is coupled with computer software for the interpretation of the IR images.

The test is ideal for detecting hot and cold spots or areas of different emissivities on the skin surface since humans radiate IR energy very efficiently.

\subsection{Science behind using Infrared Thermography to detect Breast Cancer-}

Normal tissue that is non-cancerous has a blood supply under the control of the automatic nervous system .Before a cell can become cancerous, the tissues surrounding it start to create new blood vessels.To sustain the rapid growth of these pre-cancerous and cancerous cells a constant supply of nutrients are needed. In order to maintain this supply the 'BAD' cells release chemicals in to the surrounding area, which keep existing blood vessels open, awaken dormant ones and create new ones. (This is known as angiogenesis which means new blood vessel growth)

The rich vascular beds in the breast provide the conditions necessary for growing tumor's needs. These blood vessels work hard and fast to carry nutrients to the newly formed and extremely hungry cancer cells. All that work feeding these new cancer cells produces additional heat creating "hot spots". These hot spots occur long before any tumor cells even begin to grow.

The ideal early warning system would detect both the precancerous changes occurring in the breast and the first cancer cell formations. Digital thermal imaging or infrared thermography has the ability to detect the temperature or more importantly, actually see the hot spots associated with chemical and blood vessel changes in pre cancerous as well as cancerous breast tissue. Consequently, thermography can be the first indicator that a cancer may be forming or present and in many cases from 4-10 years before it can be detected by any other method, including mammography.

\section{METHODOLOGIES \\ 3.1 Image Acquisition}

The following is a recommended set of instructions for data collection-

- Avoid use of lotion, cream, powder or deodorant on the body area to be imaged.

- Stop smoking two hours before imaging

- 7-10 minutes waiting period for thermal adaptation. The chest area to be cooled slightly with a fan

- Patient should stand at $1.5 \mathrm{~m}$ distance from the thermal camera

- Room temperature to be approximately 22 degree Celsius

- Minimize infrared source interferences. All windows must be shielded to prevent any IR radiation from coming in to the room

- Standardization of thermal imager system. An external black body source is recommended to check for any drift in the temperature sensitivity setting. The threshold temperature shall also be calibrated, as it is a reference point that the thermal imagers use to differentiate an evaluated temperature from normal temperature.
- Camera initialization- The system should be set up atleast 15 minutes before screening. Allow the scanner to stabilize with in the first 3 scans.

- Receiving three thermogram: front, right and left semi-side exposures.

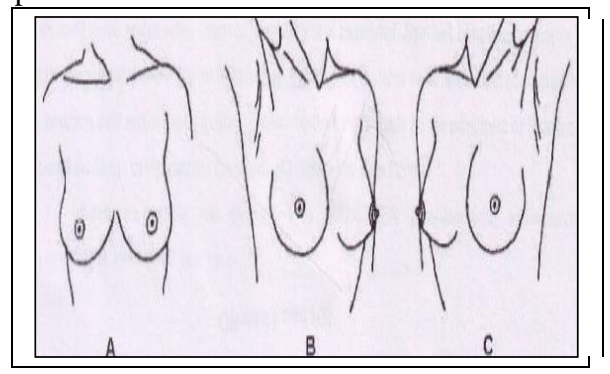

Fig2 A- Front position, B- right semi side position, c- left semi side position

\subsection{Image Processing}

Image segmentation and asymmetry analysis are proposed as an efficient method for breast analysis using infrared images. Following steps should be followed:

- Manual removal of the top body edges (e.g. shoulders) and the waistline.

- Edge detection

- Isolation of the left, right and topmost remaining body edges.

- Classify each segmented pixel into a certain number clusters

- Diagnose the breast diseases based on asymmetric analyzing of the pixels in every cluster.

\subsubsection{Edge Detection}

Edge detection is a well developed field on its own with in image processing. Region boundaries and edges are closely related, since there is often a sharp adjustment in intensity at the region boundaries. Edge detection techniques are therefore used as the base of another segmentation technique.

The edges identified by edge detection are often disconnected. To segment an object from an image however one needs closed region boundaries. Discontinuities are bridged if the distance between the two edges is within some predetermined threshold.

The built in Canrry edge detector in the Matlab Image Processing toolbox can be used to extract the boundaries of the breasts because it is one of the most precise edge detection operators and has been widely used. It is robust to noise and implements Gaussian function to smooth the image and to obtain the magnitude and orientation of the gradient for each pixel.

\subsubsection{Detection of breast boundaries:}

To find the lower boundaries of the breast is difficult because they are mostly like parabolic curves. Therefore Hough transform can be applied to extract the boundaries of the breasts. Hough transform that was proposed by Paul Hough is a method to implement a kind of mapping relation from image space to the parameter spare. The main idea can be explained as the duality between point and line. The points that in the same line in image space correspond to the intersecting lines in the parameter space. On the other hand, all the lines that intersect at a same point in the parameter space correspond to the points in the same line in image 
space. Hough transform is usually used to find the line or curve in binary map and transform the binary map into the Hough parameter calculation space.

An approach based on the Hough transform is as follows:-

- Complete the gradient of an image and threshold it to obtain a binary image.

- Specify subdivisions in the $\mathrm{\Theta}$-plane.

- Examine the counts of the accumulator cells for high pixcel concentrations.

- Examine the relationship(principally for continuity) between pixels in a chosen cell.

\subsubsection{Feature extraction for symmetry analysis of} contralateral breasts:

The features of the heat patterns may include the statistical parameters such as skewness, variation and kurtosis. The more larger the tumor size is, the steeper the temperature variation is [8]. How the temperature variates in the heat pattern is also important. Two heat patterns with the same temperature range, one may be steeper than the other for the same area has the different temperature variation or the same temperature variation occurs in the different area. The cumulative histogram which describes the temperature variation with area can reflect the asymmetry. The asymmetry can be measured by the bilatateral ratio of the quantitative features, including skewness, variation, kurtosis, temperature difference $(\Delta T)$, the maximum ratio between temperature difference and $\operatorname{area}(\Delta \mathrm{T} / \mathrm{S})$. The further the ratio from 1 , the greater asymmetry is.

The difference statistics between contralateral breasts can be used as input features to neural network analysis. The goal of the analysis would be to evaluate the suitability of ANN to assist a physician during the process of breast thermogram examinations. Fig 3 shows the block diagram for asymmetry analysis of thermogram using segmentation.

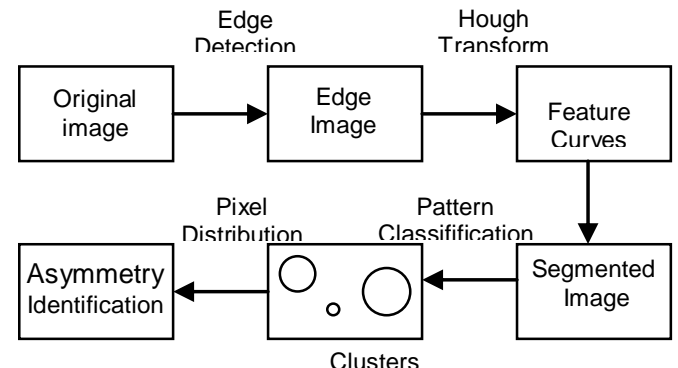

Fig 3 Block diagram for asymmetry analysis of thermogram using segmentation.

\section{Recent Advancements of Thermography -}

Modern IR real time high resolution cameras with sophisticated computers running latest image analysis software capturing 200 frames per second. It is proposed to monitor alterations in a normal vasomotor oscillation frequency band in the arterial structures of the human body as there may be disturbances in this normal oscillatory rate when a malignancy is forming.

The thermographic signal reconstruction method facilitates rapid inspection of large structures. Applications include thickness measurement of thermal barrier coatings, corrosion detection and measurement of material loss. Researchers are also trying to use the infrared thermography technique in the plastics industry for process optimization and quality improvement.

\section{CONCLUSION}

This paper proposes an approach to diagnose breast cancer based on infrared images. Firstly, the breast picture can be segmented accurately and automatically with the application of the methods of edge detection and Hough transform. Then based on pattern classification and pixel distribution asymmetries are identified for the breasts. The pathological changes of the breasts will be diagnosed based on this. The approach will be effectual and feasible and would be of great practical value in diagnosing the asymmetric abnormalities for breast using infrared images.

\section{REFERENCES}

[1] Liu Yong, Li Kai-Yang, Zhang Xian-Lin, Sun Shaeng-Rong, Wan Yin-Wen, "Analyzing method of inner heat source in breast based on infrared imaging and clinical application", 2007 Ist International Conference on Bioinformatics and Biomedical Engineering[C], ICBBE, 2007,p.853-856

[2] Cauchie Julien, Fiolet Valerie, Villers, Didier, "Optimization of an Hough transform algorithm for the search of a center", pattern Recognition [J], v 41, n2, February 2008, pp. 567-574

[3] Xujia Qin, Jionghui jiang, Weihong Wang, Zhang Fan, "Canny operator based level set segmentation algorithm for medical images" 2007 Ist International Conference on Bioinformatics \& Biomedical Engineering [C], ICBBE, 2007, pp.892-895

[4] Yu Hongshan, Wang Yaonan, "An improved canny edge detection algorithm" [J], Computer Engineering and Application, 2004, 40(20), pp. 27-29

[5] PAN Qi-ming, CHENG Yong-mei, YANG Tao, PAN Quan, ZHAO Chun-hui, "Automatic Validating and clustering method for trajectories of moving objects in real scene" [J], Application Research of computers,2007, 24(4), pp. 158-160J. Clerk Maxwell, A treatise on Electricity and Magnetism, $3^{\text {rd }}$ ed., vol. 2. Oxford: Clsrendon, 1892, pp. 68-73.

[6] M Frize, C Herry, R Roberge, "Processing of thermal images to detect Breast Cancer: A comparison," in proc. $2^{\text {nd }}$ Joint IEEE EMBS/BMES Conf., Houston, TX, pp. 1159-1160, 2002.

[7] RD Leek, "The prognostics role of angiogenesis in breast cancer," Anticancer research, vol.21, no.6B, pp. 4325-31, 2001.

[8] Sudharshan N.M., Ng E.Y.K., “ Surface temperature distribution of a breast with/without tumor," Int,J.Comput. Meth. Biomechanics Biomed. Engng.Vol.2, pp. 187-199, 1999

[9] L. Shen, R.M. Rangayyan, Desautels. J. Measures of asymmetry in mammograms based upon the shape spectrum. In Proceedings of the Annual Conf. on EMB, volume 15, pages 4849, San Diego, CA, 1993.

[10] J.Canny. A Computational approach to edge detection. IEEE Trans. Pattern Anal. and Machine Intell., 6:679-698, 1995. 